\title{
Coronal seismology using transverse loop oscillations
}

\author{
E. Verwichte ${ }^{1}$, C. Foullon ${ }^{1}$, T. Van Doorsselaere ${ }^{1}$, H. M. Smith ${ }^{2}$ \\ and V. M. Nakariakov ${ }^{1}$ \\ ${ }^{1}$ Centre for Fusion, Space and Astrophysics, University of Warwick, Coventry CV4 \\ 7AL, United Kingdom \\ 2 Max-Planck-Institut für Plasmaphysik, EURATOM-Association, D-17491, \\ Greifswald, Germany \\ E-mail: erwin.verwichte@warwick.ac.uk
}

\begin{abstract}
Coronal seismology exploits the properties of magnetohydrodynamics (MHD) in the corona of the Sun to diagnose the local plasma. Therefore, seismology complements direct diagnostic techniques, which suffer from line-of sight integration or may not give access to all physical quantities. In particular, the seismological exploitation of fast magnetoacoustic oscillations in coronal loops provides information about the global magnetic and density structuring of those loops acting as wave guides. From the oscillation period and damping time it is shown how to obtain information about the local coronal magnetic field as well as the longitudinal and transverse structuring. Furthermore, such studies motivate the development of coronal wave theories, which are also relevant for the coronal heating problem.
\end{abstract}

PACS numbers: 52.25.Xz, 52.30.Cv, 52.35.Bj

\section{Introduction}

Magnetohydrodynamic (MHD) waves occur widely in many laboratory and astrophysical plasmas where they play an important role in channeling and depositing energy (e.g. $[1,2])$. Signatures of these waves provides a tool, in conjunction with MHD wave theory, that allows to probe locally the macroscopic properties of the plasma (e.g. magnetic field, density structuring) through which they propagate. Also, they may give insight into microscopic characteristics such as transport coefficients through the examination of their damping behaviour. In the various fields of plasma research, this technique is called magnetohelioseismology in solar global interior physics (e.g. [3]), MHD coronal seismology in solar coronal physics [4], MHD spectroscopy in magnetically confined fusion [5] and magnetoseismology in space plasma physics (e.g. [6]) and in the study of accretion discs. Here, we focus on the seismological exploitation of fast magnetoacoustic oscillations in the solar corona.

The solar corona is a highly structured plasma organized into thin density enhanced magnetic flux tubes called coronal loops. The basic theory of MHD waves in coronal loops has been worked out in the 1980s (e.g. [7]). However, the technique of coronal 

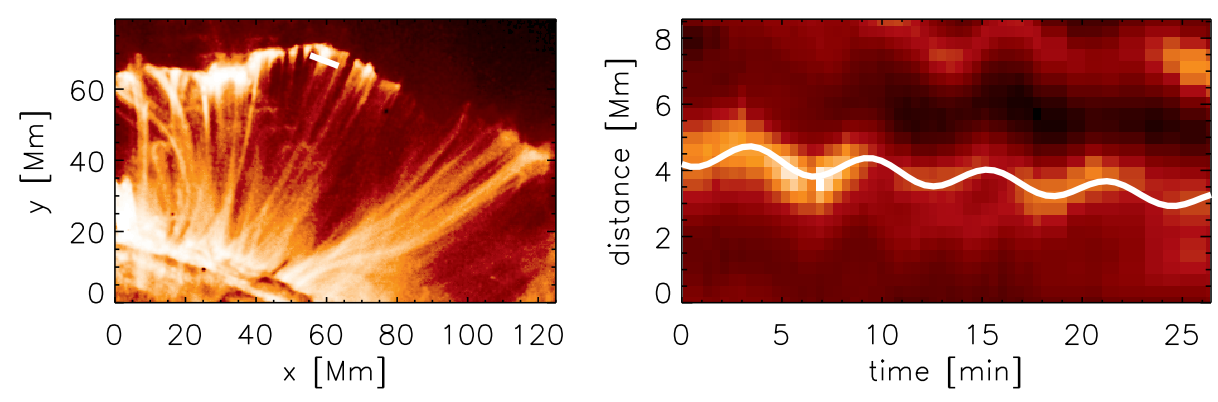

Figure 1. Left: A post-flare coronal loop arcade on the solar limb as seen by TRACE on April $15^{\text {th }}, 2001$. A linear segment is indicated that goes across a transversely oscillating loop. Right: The intensity along the linear segment as a function of time shows the TLO. A damped sinusoidal time profile fitted to the transverse location of the loop axis is overplotted.

seismology could only be put into practice after the first confident temporally and spatially resolved observations of wave dynamics became available with the launch of space missions such as the Solar Heliospheric Observatory and the Transition Region And Coronal Explorer (TRACE) in the second part of the 1990s. Through the remote detection of variations in intensity, loop position and velocity Doppler profiles, these observations revealed the presence of both slow and fast magnetoacoustic waves and oscillations in various types of coronal structures [2]. Evidence for the presence of Alfvén waves in coronal loops, where they are torsional in nature, is more difficult to obtain because of their incompressibility and incoherence [8]. Their presence is inferred through unresolved observations of non-thermal broadening of emission lines (e.g. [9]).

In particular, transverse loop oscillations (TLOs) have received much attention, i.e. large amplitude oscillations excited by a passing disturbance originating from a flaring or eruption site [10]. These oscillations manifest themselves in EUV imagers as a periodic transverse displacement of the loop axis [11] and as periodic velocity Doppler shifts in spectral instruments [12]. There is now also evidence of low amplitude transverse waves ubiquitously present in the corona [13], but they are beyond the scope of this review. Figure 1 shows a typical example of an EUV imaging observation of a TLO. The oscillation is seen as periodic transverse displacements of the loop axis. From fitting a damped sinusoidal curve to the displacement time series the oscillation signatures are determined, i.e. period, $P$, damping time $\tau$, amplitude, $\xi_{0}$. Typically, $P=2-12 \mathrm{~min}, \tau$ $=3-22 \mathrm{~min}, \xi_{0}=0.1-9 \mathrm{Mm}$ (e.g. [14]). The oscillation is dominantly the fundamental mode in the longitudinal direction but higher harmonics have also been observed [15]. TLOs have been identified as nearly incompressible fast magnetoacoustic-like kink modes [10]. Due to similarities with shear Alfvén waves in a uniform plasma, kink mode has also been called Alfvénic rather than magneto-acoustic [16]. Although TLOs are not energetic enough to explain the heating of the corona, they are nonetheless relevant. Firstly, theories put forward to explain the damping of TLOs have originally been proposed for the coronal heating problem such as resonant absorption [17]. Therefore, 
observations of transverse loop oscillations offer opportunities to verify and improve these theories. Secondly, TLOs appear in or near flaring sites and source regions of coronal mass ejections (e.g. [18, 19, 20]) and provide information about the physical conditions in such events where magnetic reconnection is thought to occur. Finally, by applying the technique of MHD coronal seismology to observations of TLOs, parameters central to coronal heating theories are derived such as the coronal Alfvén speed and magnetic field strength [21] as well as the longitudinal and transverse density structuring $[22,23]$. We shall here outline the application of coronal seismology using TLOs.

\section{Seismology of coronal loops from the TLO period}

The period, $P$, and the wavelength, $\lambda$, of a TLO can be used to deduce through the phase speed, $V_{\mathrm{ph}}=\lambda / P$, the average magnetic field strength $B$ of a coronal loop as follows. $P$ is measured from fitting a damped sinusoidal curve to the transverse displacement time series. $\lambda$ is taken from an estimate of the loop length, i.e. $\lambda=2 L / n$ with $n$ the longitudinal mode number. $V_{\mathrm{ph}}$ is compared with the dispersion relation derived from the theory of MHD waves in a low plasma- $\beta$, density enhanced, piece-wise uniform, straight cylindrical flux tube [7]. The governing wave equation for the total pressure perturbation $\hat{P}_{\mathrm{T}}(r) \sin (k z) \cos (m \phi) \exp (-\mathrm{i} \omega t)$ is of the form of a Bessel equation, i.e. $r^{2} \mathrm{~d}^{2} \hat{P}_{\mathrm{T}} / \mathrm{d} r^{2}+r \mathrm{~d} \hat{P}_{\mathrm{T}} / \mathrm{d} r-\left(\kappa^{2} r^{2}+m^{2}\right) \hat{P}_{\mathrm{T}}=0$ where in the zero plasma- $\beta$ limit the radial component of the wave vector is given by $\kappa^{2}=k^{2}-\omega^{2} / V_{\mathrm{A}}^{2}$. Continuity of total pressure and radial displacement leads to the dispersion relation for magnetoacoustic waves: $\kappa_{\mathrm{c}} n_{\mathrm{e}, \mathrm{l}}\left(\omega^{2}-V_{\mathrm{A}, 1}^{2} k^{2}\right) \mathrm{J}_{m}\left(\kappa_{\mathrm{l}} a\right) \mathrm{K}_{m}^{\prime}\left(\kappa_{\mathrm{c}} a\right)-\kappa_{\mathrm{l}} n_{\mathrm{e}, \mathrm{c}}\left(\omega^{2}-V_{\mathrm{A}, \mathrm{c}}^{2} k^{2}\right) \mathrm{J}_{m}^{\prime}\left(\kappa_{\mathrm{l}} a\right) \mathrm{K}_{m}\left(\kappa_{\mathrm{c}} a\right)=0$ where subscripts 1 and c refer to loop interior and external coronal plasma, respectively. A typical dispersion diagram for trapped modes in coronal conditions is shown in Figure 2.

In such a model the only mode that can produce significant displacements of the loop axis is the trapped kink mode (see Fig. 2). In the long wavelength limit, which is appropriate for coronal loops that have large inverse aspect ratios, the kink mode has a phase speed equal to the kink speed, $C_{\mathrm{K}}$, defined as $C_{\mathrm{K}}^{2}=\left(n_{e, 1} V_{\mathrm{A}, 1}^{2}+n_{e, \mathrm{c}} V_{\mathrm{A}, \mathrm{c}}^{2}\right) /\left(n_{e, \mathrm{l}}+n_{e, \mathrm{c}}\right)$ $\approx 2 V_{\mathrm{A}, \mathrm{l}}^{2} /\left(1+n_{e, \mathrm{c}} / n_{e, \mathrm{l}}\right)$. Thus, the phase speed is found to be proportional to the average Alfvén speed. Additional measurements of the loop density contrast and density proper allow to determine the local loop magnetic field strength. This quantity is difficult to measure directly because of the large thermal width of emission lines and the bias of radio measurements towards large magnetic field strength regions. With coronal seismology, the average coronal loop magnetic field strength is given as [21]:

$$
B=\frac{7.4}{n}\left(1+\frac{n_{e, \mathrm{c}}}{n_{e, 1}}\right)^{1 / 2}\left(\frac{L}{10^{8} \mathrm{~m}}\right)\left(\frac{P}{300 \mathrm{~s}}\right)^{-1}\left(\frac{n_{e, 1}}{10^{15} \mathrm{~m}^{-3}}\right)^{1 / 2} \mathrm{G} .
$$

The magnetic field measured using this method by several studies consistently shows a typical strength of ten to several tens of Gauss [21, 24, 25]. The application of Eq. (1) depends on accurate knowledge of loop density and contrast, which has been assumed $[21,24]$, approximated from images $[14,26]$ or measured spectroscopically [25]. The loop contrast may be included as an unknown in a self-consistent seismological method 

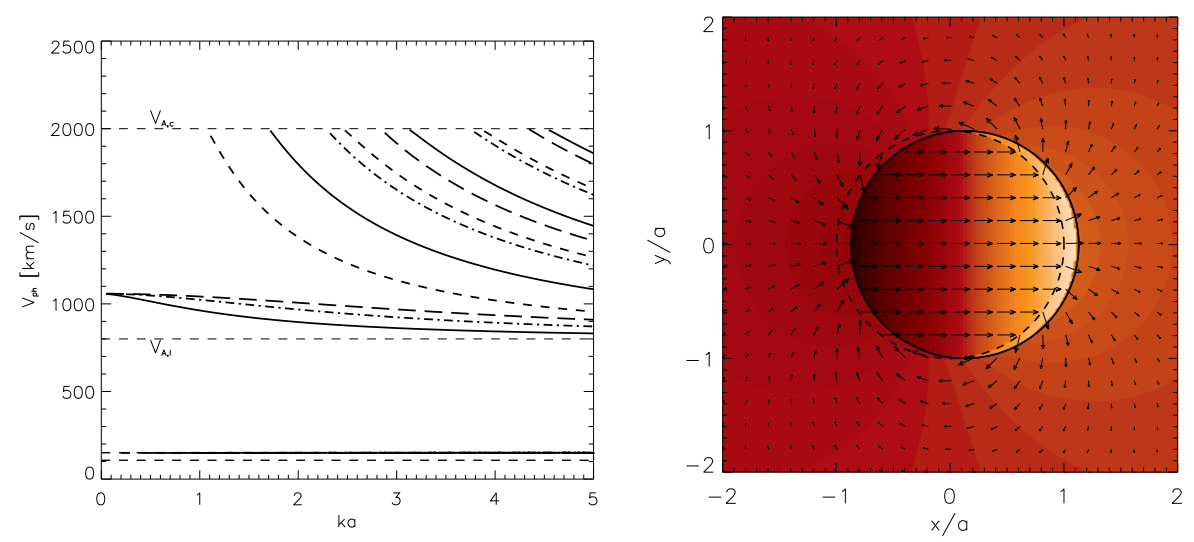

Figure 2. Left: Example of a dispersion relation for magnetoacoustic modes in low plasma- $\beta$, density enhanced, piece-wise uniform, straight cylindrical flux tube showing phase speed as a function of normalized longitudinal wave number, for four azimuthal wave numbers: $m=0$ (dashed), $m=1$ (solid), $m=2$ (dot-dashed) and $m=3$ (long-dashed). The case $m=1$ corresponds to the kink mode. $a$ is the loop radius. Right: Transverse cut through a straight uniform cylinder model of a loop for a fast kink mode. The arrows indicate the velocity field, and the color shade shows the relative density perturbation.

(e.g. [27]). Because TRACE images show the loop only in projection, the estimation of the loop length can be inaccurate as it relies on assumption of a semi-circular loop or comparison with photospheric magnetic field extrapolations. However, using data from STEREO, which observes the Sun with two space craft from two different vantage points, this source of error is reduced as the 3D geometry of the loop is obtained [26].

Furthermore, if more than one longitudinal harmonic TLO is observed simultaneously in the same loop, then the ratio of periods allows to deduce information about the density structuring and magnetic field expansion along the loop [28, 29] (see [15] for an extensive review). Such information is important to understand the equilibrium structure of coronal loops, which is governed by (unknown) heating, thermal conduction and radiative losses. For a longitudinally uniform loop the ratio of the periods of the fundamental mode with the first harmonic is approximately equal to two (there is weak dispersion, e.g. [30]). However, if the density decreases with height, as for a gravitationally stratified loop, then the period ratio becomes less than two. In contrast, a decreasing magnetic field with height (loop expansion) produces a period ratio larger than two (e.g. [32]). The few reliable observations of fundamental and first harmonic kink modes show period ratios of 1.6-1.8 [24, 25]. The density scale height is deduced from the period ratio by comparing with the theoretically predicted one found by solving the kink wave equation with a longitudinally dependent kink speed. The density scale heights are found to be in the range 30-110 Mm, compared with the gravitational density scale height of $50 \mathrm{Mm}$ for an isothermal static loop with a temperature of $1 \mathrm{MK}$. Clearly, more observations are needed before firm conclusions about the typical longitudinal equilibrium conditions can be made. 
Information about the longitudinal structuring may also be deduced from examining the spatial structure of the mode eigenfunctions themselves [31, 32, 33], if only to confirm the correct identification of the wave harmonic [24]. However, this is considerably more difficult because of the low sensitivity of the displacement profile of the fundamental mode to longitudinal structuring compared with higher overtones, and the lack of 3D information of the loop geometry and the displacement vector in single image data. As noted above, to date the 3D information of only one TLO has been extracted [26]. But even with data from two view points there remains possible ambiguity in identifying the correct harmonic, e.g. a fundamental horizontally polarized mode may resemble a first harmonic vertically polarized mode.

\section{Seismology of coronal loops from the TLO damping rate}

The characteristics of a TLO is strongly influenced by the transverse density structuring. It is after all the loop density enhancement in the first place that acts as a wave guide for fast magnetoacoustic waves. A straight piece-wise uniform density enhanced cylindrical magnetic flux tube is the simplest model that recovers the basic modes in a loop. However, it cannot explain the observed rapid damping of TLOs as classical dissipation is weak due to the nearly incompressible nature of kink modes. The transverse (radial) density structuring of the loop can contribute to the decay of the oscillation through two fundamental mechanisms: resonant absorption and wave leakage.

Firstly, a smooth continuous density profile at the edge of the loop generates an Alfvén continuum with frequencies that lie between the internal and external Alfvén frequencies. The kink mode has a frequency that lies within the continuum and has a non-zero azimuthal wave number. Hence, the global kink mode is resonantly coupled to the Alfvén continuum. This leads to local large-amplitude incoherent azimuthal motions that are damped either dissipatively or kinetically. These incoherent motions have not been observed to-date due to the lack of spatial resolution. Therefore, the kink mode is seen to damp on the time scale of the decay of the loop displacement, i.e. the timescale of the resonant mode conversion. For a edge density profile of width $\ell \ll a$, this conversion time is equal to $[34,36]$

$$
\frac{\tau}{P}=\delta\left(\frac{a}{\ell}\right) \frac{n_{e, \mathrm{l}}+n_{e, \mathrm{c}}}{n_{e, \mathrm{l}}-n_{e, \mathrm{c}}}
$$

where $\delta$ depends on the shape of the density profile, i.e. $\delta=\left[2 / \pi, 4 / \pi^{2}\right]$ for sinusoidal and linear profiles, respectively. $\ell / a$ is the thickness of the edge density profile relative to the loop radius. This result matches the observed damping times well [35] and it has been shown to be a robust result through modeling of arbitrarily wide edge density profiles, non-circular loop cross-sections and loop curvature [37, 38, 39]. In contrast, in tokamaks, fast modes are observed in the range of the ion-cyclotron frequency so that coupling to the Alfvén continuum is weak [40]. They manifest themselves as Compressional Alfvén Eigenmodes [41]. The comparison of the observed damping with the theory prediction allows to seismologically probe the transverse loop structuring [27, 44]. From comparing 

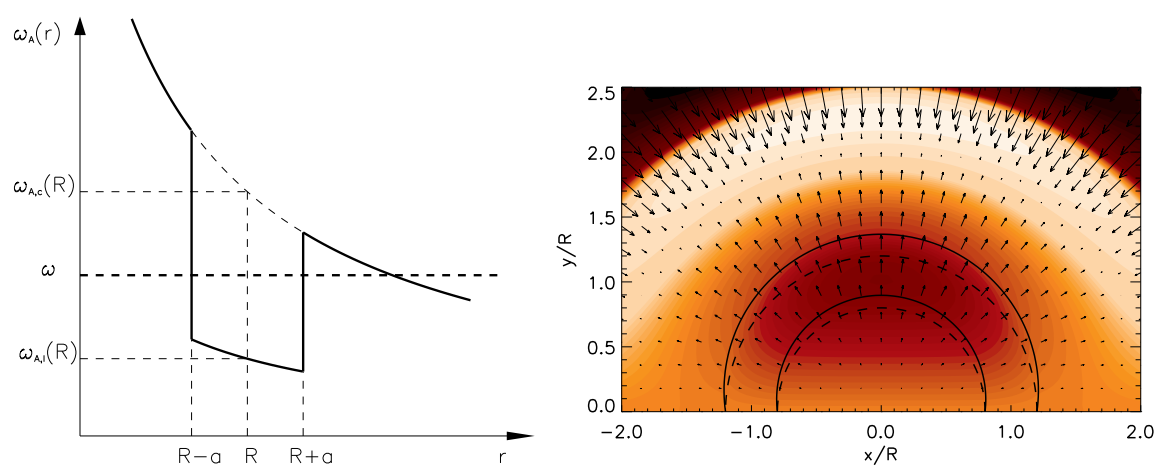

Figure 3. Left: Alfvén frequency profile as a function of distance from the origin of a semi-circular loop with potential longitudinal magnetic field. The loop has a major radius $R$ and width $2 a$. Right: Relative Lagrangian density perturbation and velocity field of a fundamental kink mode in a curved slab loop model which uses the shown transverse Alfvén frequency profile. The solid (dashed) lines show the (un)perturbed position of the loop.

self-consistently the observed with the predicted oscillation period, damping time and quality factor, information about the Alfvén speed loop density contrast and the lengthscale of the transverse density profile is determined. Thus, a lower limit for the edge density transition layer $\ell / a$ in the range $0.2-0.6$ is found.

The second damping mechanism is wave leakage into the surrounding corona due to an imperfect loop wave guide [45]. In the basic cylinder model of a loop, the external coronal plasma is assumed to be uniform with an Alfvén frequency higher than that in the loop, so that trapped modes can exist in the loop which have evanescent perturbation tails in the surrounding corona. Fast waves with frequencies above the external Alfvén frequency cannot be trapped and radiate from the wave guide (e.g. [42, 43]). Here, we focus on fast waves that are locally below the external Alfvén frequency and should be trapped but for transverse structuring. Expansion of the magnetic field and gravitational stratification of the surrounding corona as well as neighboring loops impose a varying Alfvén speed. From a certain distance the Alfvén frequency may drop below the wave frequency inside the loop and the wave perturbation becomes oscillatory once more. In this fashion wave energy can tunnel through an evanescent barrier and leak away from the loop. In a loop model that includes curvature this mechanism appears naturally because the Alfvén frequency depend on transverse (radial) distance, i.e. field strength, density and the longitudinal component of the wave vector. Figure 3 shows an example of such an Alfvén frequency profile, which shows that mode with a frequency between the Alfvén frequencies at the major radius is only trapped in the loop locally. Wave energy can tunnel through the evanescent barrier located above the loop into the surrounding corona, which results in the decay of the transverse loop oscillation [46, 47]. The wave leakage has been studied intensively analytically and numerically recently for a curved slab model, which is applicable for vertically polarized modes (e.g. [48]). Figure 3 shows the spatial structure of the kink mode, which has a considerable amount of wave energy 
above the loop in the surrounding corona. Another consequence of a varying Alfvén frequency profile is that the vertically polarized mode is no longer incompressible because the loop width does not remain constant during the oscillation. This model can be used for seismological purposes [23]. From the observed oscillation period, damping time and relative intensity amplitude, the values for the loop density contrast, the local slope of the Alfvén frequency and the Alfvén speed at the loop axis can be determined selfconsistently. This showed that the curved slab model predicts a wave leakage that is too strong to explain the observations. In fact, a slab model overestimates the wave leakage compared with a toroidal model. This can be understood using simple analytically tractable straight loop models where an evanescent barrier has been added [45]. In a Cartesian slab model, the wave solution is in terms of trigonometric functions so that wave energy decays exponentially within the evanescent barrier. In a cylindrical model where the wave solution is in terms of Bessel functions, the wave energy decays more rapidly as a modified Bessel function. Resonant mode conversion leads then to more efficient damping than due to radial radiation [49]. Preliminary numerical modeling of oscillations in a toroidal loop has shown that wave leakage is indeed reduced [50]. However, a systematic study remains to be done. Also, analytical models of a loop in toroidal geometry that includes the effect of wave leakage have not been developed yet. It is clear though that because of the continuously varying Alfvén frequency with height, wave leakage and resonant mode conversion are intimately connected, although the wave energy deposition may be outside of the loop.

\section{Discussion}

The last decade of observations of transverse loop oscillations has offered a rich testbed for MHD coronal wave theory and the application of the technique of coronal seismology. Even if these oscillations are not energetic enough to heat the corona themselves, they nonetheless are an important driver in the development of more sophisticated models that may in general be relevant to the coronal heating problem. Next generation solar missions such as the Solar Dynamics Observatory will provide a continuous stream of high resolution imaging data and make routine the application of coronal seismology as a complementary tool for diagnosing the solar plasma.

\section{Acknowledgments}

The authors wish to thank the two anonymous referees for their helpful comments.

\section{References}

[1] Wong, K.-L. 1999 Plasma Phys. Control. Fusion 411

[2] Nakariakov V. M. and Verwichte E. 2005 Living Reviews in Solar Physics 23

[3] Foullon C. and Roberts B. 2005 A $8 A 439713$

[4] Roberts B., Edwin P. M. and Benz A. O. 1984 ApJ, 279857 
[5] Goedbloed J. P., Holties H. A., Poedts S., Huysmans G. T. A. and Kerner K. 1993 Plasma Phys. Control. Fusion 35 B277

[6] Grimald S., Foullon C., Décréau P. M. E., Lerouzic G., Suraud X. and Vallières X. 2009 J. Geophys. Res. in press, doi: 10.1029/2008JA014270

[7] Edwin P. M. and Roberts B. 1983 Sol. Phys. 88179

[8] Van Doorsselaere T., Nakariakov V. M. and Verwichte E. 2008 ApJ 676 L73

[9] McClements K. G., Harrison R. A. and Alexander D. 1991 Sol. Phys. 13141

[10] Nakariakov V. M., Ofman L., Deluca E. E., Roberts B. and Davila J. M. 1999 Science 285862

[11] Aschwanden M. J., Fletcher L., Schrijver C. J. and Alexander, D. 1999 ApJ 520880

[12] Van Doorsselaere T., Nakariakov V. M., Young P. R. and Verwichte E. 2008 A\&A 487 L17

[13] Tomczyk S., McIntosh S. W., Keil S. L., Judge P. G., Schad T., Seeley D. H. and Edmondson J. 2007 Science 3171192

[14] Aschwanden M. J., de Pontieu B., Schrijver C. J., and Title A. M. 2002 Sol. Phys. 20699

[15] Andries J., Van Doorsselaere T., Roberts B., Verth G., Verwichte E., and Erdélyi R. 2009 Space Sci. Revs. in press

[16] Goossens M., Terradas J., Andries J., Arregui I. and Ballester J. L. 2009 A $\& A$, in press

[17] Hollweg J. V. and Yang G. 1988 J. Geophys. Res. 935423

[18] Verwichte E., Nakariakov V. M. and Cooper F. C. 2005 A $8 A 430$ L65

[19] Foullon C., Verwichte E., Nakariakov V. M. and Fletcher, L. 2005 A $\& A 440$ L59

[20] Nakariakov V. M., Foullon C., Verwichte E., and Young N. P. 2006 A $\& A$ 452, 343

[21] Nakariakov V. M. and Ofman L. 2001 A $\& A 372$ L53

[22] Andries J., Goossens M., Hollweg J. V., Arregui I. and Van Doorsselaere T. 2005 A\&\&A 4301109

[23] Verwichte E., Foullon C. and Nakariakov V. M. 2006 A $\& A 452615$.

[24] Verwichte E., Nakariakov V. M., Ofman L. and Deluca E. E. 2004 Sol. Phys. 22377

[25] Van Doorsselaere T., Nakariakov V. M., and Verwichte E. 2007 A $\mathscr{G} A 473959$

[26] Verwichte E., Aschwanden M. J., Van Doorsselaere T., Foullon, C. and Nakariakov V. M. 2009 ApJ 698397

[27] Arregui I., Andries J., Van Doorsselaere T., Goossens M. and Poedts S. 2007 A $\mathscr{Y} A 463333$

[28] Andries J., Arregui I., and Goossens M. 2005 ApJ 624 L57

[29] Arregui I., Van Doorsselaere T., Andries J., Goossens M. and Kimpe D. 2005 A 84444361

[30] McEwan M. P., Donnelly G. R., Díaz A. J. and Roberts B. 2006 A\&A 460893

[31] Verth G., van Doorsselaere T., Erdélyi R. and Goossens M. 2007 A $\& A 475341$

[32] Verth G. and Erdélyi R. 2008 A\&A 4861015

[33] Andries J., Arregui I. and Goossens M. 2009 A $\& A 497265$

[34] Goossens M., Hollweg J. V. and Sakurai T. 1992 Sol. Phys. 138233

[35] Goossens M., Andries J. and Aschwanden M. J. 2002 A $\& A$ A94 L39

[36] Ruderman M. S. and Roberts B. 2002 ApJ $\mathbf{5 7 7} 475$

[37] Ruderman M. S. 2003 A\&A 409287

[38] Van Doorsselaere T., Debosscher A., Andries J. and Poedts S. 2004 A $\& A 4241065$

[39] Van Doorsselaere T., Andries J., Poedts S. and Goossens M. 2004 ApJ 6061223

[40] Gryaznevich M. P. et al. 2008 Nucl. Fusion 48084003

[41] Smith H. M. and Verwichte E. 2009 Plasma Phys. Control. Fusion 51075001

[42] Zaitsev V. V. and Stepanov A. V. 1975 Issled. Geomagn. Aeron. Fiz. Solntsa 373

[43] Spruit H. C. 1982 Sol. Phys. 753

[44] Goossens M., Arregui I., Ballester J. L. and Wang T. J. 2008 A\&A 484851

[45] Van Doorsselaere T., Verwichte E. and Terradas J. 2009 Space Sci. Revs. in press

[46] Brady C. S. and Arber T. D. 2005 A $\& A 438733$

[47] Brady C. S., Verwichte E. and Arber T. D. 2006 A\&A 449389

[48] Verwichte E., Foullon C. and Nakariakov V. M. 2006 A\&A 4461139

[49] Goossens M. and Hollweg J. V. 1993 Sol. Phys. 14519

[50] Terradas J., Oliver R. and Ballester J. L. ApJ 650 L91 